\title{
REPENSANDO LA FORMACIÓN TEÓRICA A TRAVÉS DEL PRÁCTICUM: EXPERIENCIAS DE UNA DOCENTE NOVEL
}

\author{
Marcos Jesús Iglesias Martínez \\ María Moncho Miralles \\ Inés Lozano Cabezas \\ Universidad de Alicante
}

\begin{abstract}
RESUMEN: La brecha entre el saber teórico y el saber práctico está repercutiendo significativamente en la formación inicial del profesorado, aspectos fundamentales en el proceso de construcción de la profesionalización docente. Desde un enfoque cualitativo y basado en la práctica reflexiva, en este trabajo se analiza la vinculación del conocimiento teórico con las experiencias Prácticum de una maestra novel. Los resultados evidencian que el papel de la teoría sigue siendo una vertiente por la que se ha de regir exclusivamente la acción. Asimismo, se identifica que es necesario promover una relación dialógica entre el conocimiento teórico y las experiencias interdisciplinares que el Prácticum ofrece. Las principales conclusiones de este estudio demandan la creación de "espacios híbridos" (Zeichner, 2010), para mejorar la relación entre ambos conocimientos, siendo la teoría necesaria para guiar y orientar la práctica. Resulta imprescindible que la formación inicial del docente integre estos "espacios" basados en la reflexión y en el diálogo entre todos los agentes participantes, lo que permite dar sentido y significado al conocimiento teórico-práctico, con el propósito de formar profesionales capaces de observar, analizar y reflexionar las dimensiones de la enseñanza y el aprendizaje.
\end{abstract}

PALABRAS CLAVE: formación inicial docente, Prácticum, Práctica Reflexiva, contenido académico y práctico.

\section{RETHINKING THE TEACHING OF THEORY THROUGH PRACTICUMS: THE EXPERIENCE OF A PRE-SERVICE TEACHER}

\footnotetext{
ABSTRACT: The gap between theoretical and practical knowledge, both fundamental in building teacher professionalization, is significantly affecting initial teacher training. Based on a qualitative approach and reflective practice,
} 
we analysed a pre-service school teacher's conceptions and beliefs on the links between theoretical knowledge and experiences lived during the Practicum. Results showed that theory continues to represent the exclusive standpoint from which actions should be governed. Moreover, we identified the need to foster a dialogical relationship between theoretical knowledge and interdisciplinary experiences provided during the Practicum. The main conclusions of this study imply that "hybrid spaces" (Zeichner, 2010) should be created to improve the relationship between both types of knowledge, theory playing a necessary role in guiding practice. It is imperative that initial teacher training integrate these "spaces" based on reflection and dialogue between all participating agents: this will allow making sense of theoretical-practical knowledge with the aim of training professionals able to observe, analyse and reflect on teaching and learning dimensions.

KEYWORDS: initial teacher education, Practicum, reflective practice, theory and practice.

Recibido: 20/02/2018

Aceptado: 05/07/2018

Correspondencia: Marcos Jesús Iglesias Martínez, Universidad de Alicante, C/ del Aeroplano, 1, 03690 San Vicente del Raspeig, Alicante. Email: marcos.iglesias@ua.es.

\section{INTRODUCCIÓN}

La formación inicial docente desde las aulas universitarias alberga el paradigma de la relación teoría-práctica, aspecto fundamental en la construcción de una coherencia pedagógica y especialmente relevante en el proceso de profesionalización docente. El debate actual sobre la construcción de un saber pedagógico dirigido a la formación de los docentes (Dotger, 2015; Zeichner, 2014) mediante la creación de escuelas universitarias continua siendo un asunto discutido entre los teóricos de la educación (Flores, 2017; Kitchen y Petrarca, 2016), el cual ha ido generando una relevadora polisemia en lo que al significado respecta (Álvarez y San Fabián, 2013).

En este sentido, Zeichner (2010) alude a la desvinculación entre la formación universitaria y las experiencias prácticas en las escuelas como precursoras del debate entre la teoría y la práctica. Darling-Hammond (2010) insiste en la importancia de dar respuesta a los estándares de enseñanza profesional inscritos, ofreciendo oportunidades de reflexión sistemática, que superen la abstracción generada desde las aulas universitarias. La estructura del plan de estudios de la formación inicial dificulta a los estudiantes acceder a la realidad de las aulas escolares, de ahí la necesidad de que no sólo se les enseñe herramientas útiles para aplicar al aula, sino también ocasiones de ponerlas en práctica.

Korthagen (2010), bajo la propuesta del enfoque realista, sugiere un nuevo modelo aludiendo al aprendizaje a través de la experiencia, mediante la aproximación 
a situaciones reales y contextualizadas, estimulando a los estudiantes en formación a reflexionar sobre su propio pensamiento y acción, favoreciendo su desarrollo profesional como futuros docentes y contribuyendo a la construcción recíproca entre teoría y práctica educativa. Korthagen (2010) insiste en la necesidad de recurrir al saber teórico, para hacer posible una reflexión crítica que contribuya a la mejora de la práctica educativa, así como la importancia de una interacción y cooperación directa entre todas las personas que se inscriben en el proceso formativo del estudiante. En adicción, Cochran-Smith y Lytle (1999) insisten en capacitar al profesorado en formación a articular su conocimiento teórico con las experiencias vivenciadas, argumentando como a partir de la problematización de su conocimiento al entrar en contacto con un contexto real, se contribuye a mejorar su propia intervención.

Zeichner (2010), con el objetivo prioritario de conectar el conocimiento académico y práctico, considera necesario potenciar "espacios híbridos" pero, en este caso, incidiendo en la implicación de todos los miembros inmersos en la formación del profesorado, como pretexto para hacer convivir ambas perspectivas. En concreto, trata de crear una nueva forma de aprendizaje y formación del profesorado, a partir de la colaboración de docentes que trabajen en centros escolares y la transmisión y/o traslación de sus representaciones y experiencias prácticas a la formación docente universitaria de los estudiantes. De esta forma se fomenta la oportunidad de analizar y reflexionar sobre estas vertientes (conocimiento académico y práctico), beneficiándose de ambas fuentes en la construcción de su pensamiento como docente. Todo ello, mediante la creación de lugares de encuentro entre profesionales de la educación: formadores universitarios, profesionales de la enseñanza en centros escolares y estudiantes en su formación inicial como docentes.

El objetivo de este trabajo pretende analizar la relación entre conocimiento teórico y práctico en la formación inicial docente a través de la propia experiencia de una maestra novel. La docente analiza y vincula, mediante la reflexión, la conexión entre ambos ámbitos.

\section{La Práctica Reflexiva como medio para promover la conexión entre LA TEORÍA Y LA PRÁCTICA}

Los planes de estudio de formación inicial del profesorado son los responsables de diseñar y establecer puentes intermedios para favorecer la vinculación del conocimiento teórico (impartidos en las aulas universitarias) con el conocimiento práctico (desarrollados a través del Prácticum). En este sentido, Álvarez y Hevia (2013), González y Fuentes (2011) y Zabalza (2016) coinciden en considerar el Prácticum como uno de los elementos clave en la formación inicial docente, un momento predilecto para interrelacionar teoría y práctica, utilizar y reformular lo aprendido a través de la reflexión (Domingo y Gómez, 2014; Rodgers y LaBoskey, 2016). El Prácticum permite reflexionar sobre la experiencia práctica y continuar construyendo el pensamiento y la identidad del docente, otorgando significado al conocimiento académico, tal y como afirman González y Fuentes (2011): "como estudiantes acostumbrados a asimilar y reproducir conocimiento teórico, experimentan la urgencia de resituar lo que saben, contextualizarlo y movilizarlo para orientar sus percepciones (...)" (p.57). 
El Prácticum se aproxima al modelo de "espacios híbridos" que Zeichner (2010) propone, ya que proporciona la oportunidad de interactuar con diversos agentes educativos inscritos en contextos diferentes, el/la tutor/a de la facultad desde las aulas universitarias, y el/la tutor/a del centro desde las aulas escolares; percibiendo así las posibilidades que ambas vertientes (teoría y práctica) ofrecen. Los escenarios profesionales reales como son las aulas escolares, permiten al alumnado iniciarse como docentes (Zabalza, 2016), potenciando la Práctica Reflexiva (Domingo y Gómez, 2014; Rodgers y LaBoskey, 2016) como una competencia más de los docentes, fomentando la posibilidad de profesionales que investigan sobre su propia acción (Day, 2005). Se trata, por tanto, de dar paso a la reflexión e indagación desde la formación inicial del profesorado para llegar al saber resultante de la práctica profesional como docentes reflexivos (Perrenoud, 2004; Schön, 1992).

Es necesario, por tanto, una reconceptualización del conocimiento práctico actual (Clarà y Mauri, 2010) en el que se integren el diálogo y la reflexión entre ambos conocimientos como análisis del discurso en la formación del profesorado, y que enfatizan el significado de cómo un docente aprende a reflexionar y a investigar sobre las propias situaciones del aula (Cochran-Smith y Villegas, 2015) para reconocer las posibilidades de producir y construir conocimiento en la práctica.

En síntesis con lo expuesto, el propósito del presente trabajo es analizar y delimitar el paradigma de la relación subyacente entre teoría y práctica en la formación inicial docente, mediante un ejercicio de Práctica Reflexiva (Domingo y Gómez, 2014). Por consiguiente, las cuestiones que orientan y fundamentan esta investigación son las siguientes:

- ¿Qué posibilidades interdisciplinares ofrece el Prácticum en la búsqueda de una dualidad entre conocimiento y acción en la formación como maestros/as?

- ¿Qué aportaciones realiza la formación de profesionales reflexivos de su propia práctica?

\section{Diseño y METOdología DE LA INVESTIGACIÓN}

Se opta por una metodología cualitativa a través de un estudio de caso único que permite el análisis de las experiencias de los docentes en su formación (Huber, Caine, Huber y Steeves, 2013). Se utiliza la estrategia cualitativa autobiográfica narrativa y reflexiva (Bolívar, 2017; Kelchtermans, 2014) de una maestra que ha finalizado sus estudios de Grado en Educación Infantil. La docente ha pasado por un proceso de aprendizaje teórico-práctico, complementado con estrategias de la Práctica Reflexiva (Domingo, 2013) así como con técnicas documentales características de la formación inicial del Grado en Maestro/a en Educación Infantil (Fleet, Patterson y Robertson, 2017). En este sentido la participante elabora, de manera voluntaria y durante un periodo de cuatro meses, un ensayo reflexivo sobre la relación de la teoría de tres asignaturas del área de Didáctica y Organización Escolar con las vivencias en el Prácticum. La elección de esta maestra tiene un carácter intencional no probabilístico en base a las características de la investigación cualitativa. En esta elección ha participado el equipo investigador constituido por seis docentes 
del área de Didáctica y Organización Escolar que Ileva desarrollando la línea "La relación teoría-práctica en la formación inicial en las asignaturas de Didáctica y Organización Escolar" durante cinco cursos académicos. El propósito de esta línea es orientar y formar al alumnado del Grado en Maestro/a sobre la reflexión entre el saber teórico y práctico. En este sentido el ensayo reflexivo elaborado por la participante ha sido seleccionado en base a tres criterios: calidad argumentativa y reflexiva, coherencia y articulación en el desarrollo del ensayo, y fundamentación teórica utilizada para el ensayo reflexivo.

Para el análisis del ensayo reflexivo de la maestra se utiliza el programa cualitativo AQUAD 7 (Huber y Gürtler, 2012), soporte informático que garantiza la codificación de los resultados cualitativos. En este proceso se conjuga la interacción entre los códigos provenientes de los relatos de la participante y la conceptualización y estructuración del mapa de códigos emergentes discutidos por el equipo investigador y que han sido agrupados en tres temáticas principales. En este sentido, los códigos resultantes han sido validados por tres expertos en investigación cualitativa, que a su vez son investigadores y docentes en el área de Didáctica y Organización Escolar.

\section{Resultados}

En el proceso de codificación de las experiencias personales referentes a la relación teórico-práctico los resultados se han agrupado en tres temáticas referidas a: (T1) los procesos en el diseño y desarrollo del currículo; (T2) el ambiente de aprendizaje en el aula; (T3) las interrelaciones de comunicación docente. En la presentación de resultados se sigue el hilo conductor de las temáticas, para ello se indica al final de cada narrativa el número de la temática y el código emergido: (Narrativa T1. Código)

\section{Temática 1. Los procesos en el diseño y desarrollo del currículo}

Con respecto a la planificación de la intervención en el aula, en este caso de una propuesta didáctica seleccionada por la participante, se describen experiencias referidas al diseño y puesta en práctica de las Unidades Didácticas (contenidos, metodología, tiempo, espacio, etc.) desarrolladas en los periodos de prácticas. En este sentido, la maestra resalta las diferentes modificaciones o alteraciones referidas, en concreto a los elementos de carácter metodológico y organizativo, lo cual supone la necesidad de revisar y reorganizar la distribución de las sesiones:

En mi caso, fue a partir de la estructuración y distribución temporal de mi propuesta didáctica, previamente con una secuenciación de, aproximadamente, quince actividades más aisladas, y un desarrollo temporal que se extendía dos semanas, donde experimenté la necesidad de revisar y reorganizar la distribución, tanto didáctica, como temporal previamente programada. Fue realmente al acceder al interior del aula cuando pude comprobar la conexión con la teoría. Me di cuenta de cómo la dinámica de aprendizaje de ésta respondía a un tratamiento más globalizado, y se caracterizaba por trabajar por sesiones, integrando en cada una de éstas tres tipos de actividades y/o fases (inicial, 
de desarrollo, y de síntesis), e, igualmente, el ritmo de aprendizaje del alumnado estaba acostumbrado a ello. En este punto el contenido teórico me ayudo mucho. (NarrativaT1. Programación)

Esta investigación permitió a la participante reflexionar acerca de la influencia que ejercen múltiples variables en la implementación de la programación, repercutiendo también en el diseño previo de ésta y la necesidad de conocer y familiarizarse con las características que definen el contexto en el que se inscribirá la acción e intervención de los futuros docentes:

Modifiqué mi organización programada articulándolas y agrupándolas al esquema de una secuenciación por sesiones más afín al contexto del aula. Estas circunstancias siempre fueron comentadas en las aulas universitarias. (NarrativaT1. Modificaciones programación)

Asimismo, la maestra destaca la necesidad de un espacio y situación contextualizada, revelando la significación de acceder a la práctica y así nutrir el saber teórico, siendo éste también imprescindible en la mejora e innovación de la práctica educativa, tal como se indica en este extracto:

Durante el conocimiento teórico cultivado a lo largo de mi formación inicial docente, pude ofrecer una respuesta ajustada al aula en la que se iba a focalizar mi intervención, siendo, por ejemplo, en dos de las asignaturas de Didáctica y Organización Escolar donde se me transmitieron contenidos vinculados con la programación y organización de actividades por sesiones, permitiéndome así poseer un bagaje que me ayudó satisfactoriamente en lo que se refiere a la innovación. (NarrativaT1. Innovación)

Además de las situaciones del contexto y de los tiempos en los que se desarrolla el proceso didáctico, la docente refiere la importancia del ritmo de aprendizaje del alumnado que va ligada a la educación emocional para llevar a cabo las propuestas didácticas. Sin embargo, este aspecto no es descrito por la maestra como un contenido tratado en la teoría:

El ritmo de aprendizaje del alumnado [...] A la hora de sumergirse en la educación emocional, una competencia que requiere tiempo y debía de ajustarse a sus capacidades [...] El miedo y la calma fueron emociones que no pudieron trabajarse de la misma forma que las otras tres emociones básicas. (NarrativaT1. Otros contenidos)

La finalidad de la evaluación en la Educación Infantil es otro de los elementos que destaca la maestra en su proceso de reflexión. En este caso van dirigidas expresamente a la evaluación del aprendizaje por parte del alumnado, tanto de sus conocimientos previos respecto al hilo conductor de la propuesta didáctica programada, como los resultados finales del aprendizaje de la misma. En este sentido la participante enfatiza que a lo largo de su formación inicial se les ofrecen distintos sistemas de evaluación, pero sin una vinculación directa con la práctica:

Es cierto que desde las aulas universitarias se nos invita a la utilización de un portafolio o cuaderno de aprendizaje a modo de seguimiento de la asignatura, pero no se nos enseña 
ni se crean situaciones para familiarizarnos con dicho instrumento de evaluación y las posibilidades de ponerlo en práctica en la realidad de las aulas. (NarrativaT1. Evaluación)

Por otra parte, la docente destaca que pudo comprender algunas estrategias innovadoras de evaluación utilizadas por la tutora de su aula. Es el caso de la técnica de evaluación basada en la observación activa y participativa, que permite la evaluación integral en el proceso educativo:

Gracias a lo que había aprendido en la universidad pude identificar y comprender una estrategia innovadora de evaluación utilizada por mi tutora: la evaluación compartida. Me ayudó a conocer las opiniones de los niños/as en relación con lo que habían aprendido y qué era lo que más les había gustado o llamado la atención, otorgándome la facultad de valorar mi intervención, reflexionar acerca de los aspectos a mejorar..., e implicando más significativamente a los niños/as en la evaluación de los aprendizajes. De forma que, el docente del aula, a la hora de evaluar, dedicaba, secuencialmente, momentos de la jornada escolar dirigidos a valorar conjuntamente el objeto y proceso de enseñanza-aprendizaje (¿qué hemos aprendido?), es decir, a poner en práctica la evaluación compartida. (NarrativaT1. Evaluación)

La interconexión entre la teoría y la práctica potencia en los docentes un proceso de autoevaluación en el diseño y desarrollo del currículo, aspecto con el que concluye la participante en relación a la dimensión planificación de la docencia:

Nos permite prever y vaticinar posibles alteraciones que incidirán en nuestra intervención y actuación docente, brindándonos la oportunidad de corregirlas previamente al inicio de la puesta en práctica, favoreciendo nuestra reflexión sobre la propia práctica docente. (NarrativaT1. Autoevaluación)

\section{Temática 2. El ambiente de aprendizaje en el aula}

El saber teórico proporciona una primera aproximación conceptual respecto a cómo gestionar un ambiente de aprendizaje, pero de una forma generalizada que, en ocasiones, no coincide con lo que posteriormente se observa en los centros y las aulas escolares. En la siguiente narrativa se constata la distancia entre el saber y el hacer:

El agrupamiento del alumnado fue una de las diferencias que más noté con respecto a la teoría. Mi tutora recurría a un agrupamiento del alumnado, en pequeño grupo (7-8 niños/as), de un total de 24 niños/as, para el desarrollo semi-dirigido de conceptos vinculados con conocimiento matemático. El planteamiento metodológico del docente residía en la puesta en práctica de talleres, de carácter heterogéneo, ofreciendo así un apoyo y atención más individualizada a cada niño/a, favoreciendo el aprendizaje fundamentado en la socialización entre iguales, dado que al agruparse niños/as con diversidad de capacidades, se les ofrecía la posibilidad de autocorregirse autónomamente, así como de ejercer un papel de tutorización, y ser partícipe de un aprendizaje recíproco. (NarrativaT2. Agrupación) 
El conocimiento teórico aporta información referente a las oportunidades pedagógicas de la planificación de rincones y talleres, así como su funcionalidad y los criterios para su correcto funcionamiento. Desde la formación inicial se pueden proponer actividades variadas en cada uno de estos rincones, pero no en un contexto real, sino mediante un caso práctico o situación idílica que puede no corresponderse con la realidad del aula. En cualquier caso, las prácticas suponen una oportunidad para profundizar en otros aspectos en lo que se refiere la distribución de estos espacios para el desarrollo de las áreas de conocimiento:

Tuve la oportunidad de observar y atribuir significado a los dilemas constructivos que se le presentaban a el docente a la hora de organizar y gestionar el espacio del aula. Por ende, el rincón y/o zona de trabajo era aprovechado, en ocasiones, para la puesta en práctica de talleres temporales (de letras, de plástica, de lógico-matemática, de experimentación...), siendo el caso del rincón de la asamblea un enlace entre actividades, dado que cada niño/a poseía un ritmo diferente de aprendizaje. No obstante, reflexionando sobre su propia práctica, basándose en anteriores experiencias, con la intención de no perder la oportunidad que se les ofrecía a los niños/as de acceder a un muestrario de libros, cultivando su gusto por la literatura, y el inicio del hábito lector, el docente decidió mantener dicha parte del espacio, aportando material didáctico vinculado con el centro de interés pertinente. (NarrativaT2. Distribución contenidos curriculares en el espacio)

El ambiente de aprendizaje también recoge otras experiencias muy representativas referidas a la selección de los recursos y materiales a utilizar en el aula. En este sentido, desde las aulas universitarias se incide en la necesidad de atender a unos criterios en la selección de los materiales como recurso didáctico, requisito para garantizar el éxito en su funcionalidad respecto a los aprendizajes propuestos:

La teoría nos aproxima a los aspectos en los que tenemos que centrar nuestra atención a la hora de seleccionar el material didáctico, que guiará nuestra práctica docente, pero resulta necesario nutrirnos de una aproximación al contexto real en el que se delimita nuestra acción, para poder construir un ambiente favorable de aprendizaje gracias a las características propias de cada aula. (NarrativaT2. Recursos-materiales)

\section{Temática 3. Interrelaciones de comunicación docente}

Los procesos de comunicación educativa están estrechamente vinculados con las interacciones interpersonales e intrapersonales entre el alumnado, el profesorado y los familiares. En esta temática se incluyen las experiencias que potencian la comunicación del docente con los agentes educativos y de esta manera conocer e identificar esas relaciones verticales y horizontales en el contexto educativo.

En las aulas universitarias se desarrollan una variedad de contenidos conceptuales y procedimentales sobre situaciones prácticas simuladas que implican la observación de las interacciones sociales en las aulas. De esta forma, los docentes en formación adquieren contenidos actitudinales y valores asociados a esas relaciones sociales, pero es 
difícil aproximarse al desarrollo socio-afectivo de los niños/as fuera del contexto real. En este sentido, en la teoría se destaca la característica principal de la asamblea como lugar de encuentro y acogida de experiencias, lo cual fomenta las relaciones sociales gestionando la interacción horizontal (entre iguales) de los niños/as, ofreciendo así ocasiones de análisis y discusión en relación con los conflictos, lo que permite identificar las distintas estrategias comunicativas que se han de emplear para cada situación:

Principalmente, mi tutora se hacía servir, tanto en la jornada escolar matinal, como vespertina, de la asamblea como lugar de encuentro, dirigida al desarrollo de conductas sociales, a aprender a escuchar, rompiendo con el estereotipo de atribuir al significado de asamblea el trabajo de las rutinas diarias, convirtiéndose en un espacio que invitaba a indagar en los conflictos, sentimientos y/o emociones del alumnado. Por primera vez pude observara y experimentar estas características que estudié y analicé en la asignatura de Organización del Aula. (NarrativaT2. Interacción social alumnado)

Por otra parte, también se da importancia a la educación emocional como otra de las características principales de la asamblea como espacio de encuentro, diálogo y reflexión:

Brindaba la posibilidad a cada niño/a de expresar cómo se sentían, y por qué se sentían así, y si le podían ayudar de alguna forma, indagando en el interior de cada uno de ellos/as; en este aspecto los compañeros/as proponían soluciones para ayudar a éste/a a mejorar su estado de ánimo. (NarrativaT2. Interacción social alumnado: emociones)

La familia constituye el agente socializador por excelencia, por lo que, resulta necesario vivenciar experiencias vinculadas con la interrelación y comunicación familia-escuela, otro de los factores presentes en la formación teórica del docente:

En mi centro proponían a los familiares que se animaran a trasladar al aula alguna actividad, de la que pudieran disfrutar, tanto el colectivo discente, como el docente. Como resultado, algunas de las familias ponían en práctica actividades (participación sistemática) vinculadas con talleres de animación a la lectura o incluso de experimentación y manipulación, como la mesa de luz; actividades de las que se pueden derivar significativos beneficios, tanto a nivel de favorecer la participación e interrelación familia-docente. [P]or cierto, un contenido muy trabajado en la universidad. (NarrativaT3. La familia en el proceso de E/A)

Igualmente, otra de las estrategias observadas por la participante es la utilización de las TIC para el fomento de la interrelación familia-escuela:

Era compartir, mediante un blog, todas las actividades que, progresivamente, se iban realizando en el aula, y de esta forma, implicar a las familias en el proceso de enseñanza-aprendizaje de sus hijos/as de una manera más próxima, mediante la visualización de fotografías reales, aspecto que podía estimular la creación de momentos entre progenitores e hijos/as, incrementando así el intercambio oral mutuo. Esta estrategia la estudié en una asignatura Grado. Pude comprobar que sí resulta beneficiosa para la comunicación entre familia y escuela. (NarrativaT3. La familia y las TIC) 
Cada docente posee un pensamiento pedagógico propio fruto de sus experiencias. Sin embargo, el conocimiento docente se va construyendo a partir de la interrelación con otros/as compañeros/as que, posiblemente, hayan sido formados partiendo de un modelo diferente o compartan otras concepciones pedagógicas, que pueden interactuar favorablemente y así ofrecer un proceso de enseñanza-aprendizaje con la mejor calidad posible. En este sentido, la participante resalta la importancia de la coordinación entre los docentes, pues supone una observación directa acerca de las reflexiones dialógicas y compartidas que hacen de su práctica diaria:

En la asignatura Diseño de los Procesos Educativos de la carrera se abordó la importancia del trabajo colaborativo entre los docentes. En este sentido, tuve la posibilidad de asistir a una reunión de ciclo, mediante la cual tomé conciencia de la necesidad de una comunicación y coordinación periódica entre los agentes de la comunidad educativa. Atendiendo a dicha experiencia, se incidía significativamente en la conformidad del ciclo de cara al funcionamiento de la evaluación a lo largo de los trimestres correspondientes, cuestionándose si los instrumentos y/o herramientas utilizadas eran las adecuadas, o la necesidad de considerar la introducción de alguna propuesta de mejora. Por consiguiente, dicha experiencia me permitió reflexionar y valorar la necesidad de una confluencia de las responsabilidades entre los agentes educativos, dado que constituye un momento de reflexión del equipo docente sobre su propia práctica, su propia acción, y el cómo, exponiendo diferentes puntos de vista, pueden modificar, mejorar e innovar en su intervención. (NarrativaT3. Coordinación y colaboración el equipo docente)

\section{Discusıón}

En lo que se refiere a la importancia de programar y gestionar previamente cada uno de los elementos que constituyen una propuesta didáctica, la maestra novel verifica la dificultad que supone programar una Unidad Didáctica o Proyecto. La docente afirma que es necesario modificar la secuencia temporal e incluso actividades que no llegan a realizarse. Estas situaciones fueron indicadas durante la formación teórica, sin embargo su compresión no fue hasta el momento de las prácticas, aspectos que coindicen con el estudio presentado por Saiz y Susinos (2017) y en la investigación descrita por Stenberg, Karlsson, Pitkaniemi y Katriina-Maaranen (2014).

La evaluación también es considerada como un elemento clave y primordial en el diseño, la planificación y la futura acción docente. Además la participante considera que el hecho de realizar la tarea de evaluación no solo supone conocer si el alumnado ha adquirido o no las capacidades, sino que favorece el proceso de reflexión docente, tal como han indagado Mena, Sánchez y Tillema (2011) demostrando que las prácticas válidas son las basadas en experiencias y evaluaciones reales, con el propósito de crear situaciones y momentos de retroalimentación en relación al conocimiento transmitido y generado (Gessa, 2011). La reflexión sobre la propia acción se concibe como ese puente intermedio favorecedor de la relación entre saber y hacer (Álvarez, 2012), aspecto enfatizado por la maestra. Estos métodos también se inscriben en la innovación docente en el Prácticum (White y Forgasz, 2016), es- 
pecialmente, es considerado en los contenidos teóricos como elemento que puede contribuir a la calidad de la enseñanza y el aprendizaje, componente destacado por Darling-Hammond (2017).

En el ambiente de aprendizaje, la maestra destaca la oportunidad de conocer aspectos vinculados con la planificación y organización de éste, principalmente, en relación a la importancia de distribuir el espacio en talleres y rincones como elementos integradores del currículo. En este sentido se afirma que el saber teórico proporciona una primera aproximación conceptual respecto a cómo gestionar un ambiente de aprendizaje, pero de una forma generalizada y mediante supuestos prácticos. Se trata, por tanto, del conocimiento en acción, la reflexión sobre la propia práctica y el ensayo-error (Shön, 1992). Se pasa de un conocimiento fruto de la teoría y la lógica, a un conocimiento que se genera espontáneamente en interacción con la práctica educativa, ya que a través del saber teórico se desconocen aspectos fundamentales a tener en cuenta, los cuales varían de un ambiente a otro como, por ejemplo: la ratio de alumnado, los materiales disponibles, el mobiliario y dimensiones del aula, entre otros. Esto supone una muestra más de la necesaria conexión entre teoría y práctica, problemas pedagógicos que han sido evidenciados por Saiz y Susinos (2017), como elementos que permiten cuestionar y reflexionar la práctica educativa de los futuros docentes.

La mayoría de los modelos de formación inicial del profesorado defienden la importancia de crear comunidades docentes participativas y colaborativas. En este sentido, los relatos reflexivos descritos por la maestra destacan la importancia de las interrelaciones comunicativas entre los distintos agentes educativos (docentes, alumnado, familia, formadores etc.) y las cuales pueden considerarse como Comunidad de Aprendizaje en la que se construyen significados e identidades cambiantes entre los distintos formadores a través del diálogo y la reflexión, atributos que también se han demostrado en el estudio de Williams (2014). El Prácticum tal y como señala Álvarez (2013), ofrece la posibilidad de reconstruir y reflexionar sobre lo aprendido. La interacción y el hecho de compartir con otros agentes educativos estas prácticas, permite tomar conciencia de otras posibilidades, derivándose nuevas habilidades y destrezas sociales para los docentes en formación (Álvarez y San Fabián, 2013). Este tipo de observaciones, realizadas por la maestra novel, han supuesto un aprendizaje de las estrategias basadas en la reflexión dialógica y colaborativa como parte de una comunidad de aprendices que enriquece su formación gracias a la experiencia en una comunidad que aprende en conjunto. En este sentido, los relatos de maestra son coherentes con los componentes destacados por Glass y Rud (2012) en el que el diálogo, la comunicación, la interacción y la relación con otros miembros de la comunidad educativa permiten reflexionar y cuestionar sobre algunas teorías y modelos que se analizan y estudian en las titulaciones universitarias.

\section{CONCLUSIONES E IMPLICACIONES}

El objetivo principal de este trabajo ha sido delimitar y analizar la relación teoría y práctica educativa subyacente en la formación inicial del profesorado a través de los relatos de vida de una docente novel. La teoría, hasta el momento, ha ido adqui- 
riendo un papel prioritario frente a la práctica, mayoritariamente subordinándose a ésta. El análisis de las reflexiones elaboradas por la maestra participante nos permite concluir que la teoría aún requiere una vinculación directa con la práctica educativa. Es por ello necesario una reconceptualización de los modelos de formación docente (Lieberman y Pointer-Mace, 2008), pues el conocimiento teórico ha de ser un instrumento complementario que ayude a comprender e interpretar la práctica docente. Resulta imprescindible que la formación inicial, desde un primer momento, tengan cabida experiencias prácticas, que aproxime a la contextualización del aprendizaje, percibiendo las desviaciones y transformaciones al acceder a un contexto real en las que se producen; por ejemplo, atendiendo a las experiencias referidas al diseño y planificación de la programación de aula o al ambiente de aprendizaje y, de esta manera, partiendo de la construcción de una teoría vivenciada (Whitehead, 2009, citado por Álvarez, 2013). Igualmente, esta conexión dialógica entre teoría y práctica, también permite ir construyendo la identidad de los futuros docentes en prácticas (Álvarez, 2012), así como cuestionar tanto el planteamiento teórico como el práctico.

Del mismo modo, respecto a la influencia que ejerce la creación de "espacios híbridos" (Zeichner, 2010) y/o comunidades profesionales de aprendizaje (Dufour, 2004; Green, Ruutz, Houghton y Hibbins, 2016; Wenger, 2001) con identidad de respuesta a un aprendizaje dialógico, ésta se inclina a favorecer notoriamente la noción de aprendizaje contextualizado, es decir, permite romper con la desconexión entre el conocimiento teórico más imperante en la formación inicial desde las aulas universitarias, frente al conocimiento práctico fruto del acceso a un contexto próximo al ejercicio profesional docente, ampliando el pensamiento y las creencias hacia formas más complejas de conocimiento. Actualmente, la idea de "espacios híbridos" empieza a inscribirse en las aulas universitarias (Payne, y Zeichner, 2017), como forma de vincular a los docentes en formación inicial con su desarrollo profesional, como una manera de realzar el papel de la práctica, pero, en este caso, con un carácter social y de retroalimentación del saber teórico y viceversa. No obstante, tras analizar e interpretar los resultados obtenidos, el Prácticum resulta susceptible de concebirse como una primera aproximación a la idea de Zeichner (2010), donde se produce un encuentro interdisciplinar entre el alumnado en prácticas, un equipo docente ejerciendo como profesionales en un centro educativo y el/la tutor/a de la Universidad. El trabajo colaborativo y reflexivo en este espacio puede garantizar efectos positivos en la formación inicial del profesorado (Bozu y Aránega, 2017).

Sucintamente, se ha podido constatar que el Prácticum constituye una oportunidad para cuestionar el conocimiento didáctico, atribuirle significado en relación con las demandas sociales a las que se enfrentaran los futuros docentes, familiarizándose con otras competencias y/o dimensiones del profesorado no adquiridas desde las asignaturas universitarias. Igualmente, brinda la oportunidad de asumir un rol más activo en lo que al ejercicio de enseñar respecta, interiorizar actitudes más próximas al rol profesional docente, dentro de un contexto socioeducativo real (González y Fuentes, 2011), a reflexionar sobre la acción para comprenderla mejor y ayudar a buscar coherencia y cohesión entre lo que se sabe, se hace y se piensa. Del mismo modo, el Prácticum genera la necesidad de ejercer un tratamiento globalizado de los contenidos que han constituido, hasta el momento, el saber teórico, es decir, resulta fundamental 
una convergencia entre los contenidos de las diferentes asignaturas, para poder ofrecer respuestas adecuadas al contexto en el que en ese periodo se inscribe. El Prácticum sirve de tamiz para valorar el papel de esa "cultura técnica" (Day, 2005) que se construye desde la universidad, pero ahora en diálogo constante con la realidad práctica y social.

Finalmente, el aprendizaje académico universitario estimula a reflexionar sobre la importancia de contenidos teóricos que se transmiten, o incluso sobre la realización de ejercicios prácticos, pero todavía se conciben lejos del futuro escenario profesional (las aulas escolares); tratándose de una reflexión académica concentrada en los contenidos y el modo de enseñarlos. Principalmente, la Práctica Reflexiva (Domingo y Gómez, 2014) constituye una metodología y/o herramienta que ha de aprenderse. Esta estrategia formativa puede resultar compleja para el profesorado en formación, pero el Prácticum concede un significado personal a esos contenidos transmitidos (Álvarez, 2013). Así cabe también destacar que la formación inicial desde las aulas universitarias se incide en la adquisición de competencias para forma profesionales docentes reflexivos (Perrenoud, 2014), es decir, ser conscientes del verdadero significado de la enseñanza y del aprendizaje y de sus aspectos implícitos, a los que reflexionando académicamente es posible conocer las necesidades y demandas sociales del contexto en el que estribará el futuro ejercicio docente. Todo ello a través de la autobservación y el desarrollo de juicios críticos, ya que permitirá al futuro docente interpretar la acción educativa e innovar sobre ella en busca de la calidad. No obstante, para ello necesitamos reivindicar esa relación dialógica entre teoría y práctica, donde ambas se construyan mutuamente, es decir, sea posible la reflexión sobre la propia acción educativa. En este sentido, dicha brecha entre ambos conocimientos constituye el reflejo de un distanciamiento entre ambas instituciones. Es por ello necesario que cada una de éstas trabaje de manera colaborativa e interdisciplinar para garantizar la calidad de la formación inicial del profesorado.

\section{REFERENCIAS BIBLIOGRÁFICAS}

Álvarez, C. (2012). La relación teoría-práctica en los procesos de enseñanza-aprendizaje. Educatio Siglo XXI, 30(2), 383-402.

Álvarez, C. (2013). ¿Puede superar el profesorado la quiebra teoría-práctica? Un estudio de caso. REICE. Revista Iberoamericana sobre Calidad, Eficacia y Cambio en Educación, 11(4), 109-127.

Álvarez, C. y Hevia, I. (2013). Posibilidades y límites de la relación teoría-práctica en la formación inicial del profesorado. Cultura y Educación, 25(3), 337-346.

Álvarez, C. y San Fabián, J.L. (2013). Perspectivas para comprender la relación entre la teoría y la práctica en la formación del profesorado. Enseñanza \& Teaching, 31(1), 23-38.

Bozu, Z. y Aránega, S. (2017). La formación inicial de maestros y maestras a debate: ¿qué nos dicen sus protagonistas? Profesorado: Revista de Currículum y Formación del Profesorado, 21(1), 143-163.

Bolívar, A. (2017). Biographical and narrative research in iberoamerica: Emergence, development and state fields. En I. Goodson, A. Antikainen, P. Sikes y M. Andrews 
(editors). The Routledge International Handbook on Narrative and Life History (pp. 202-213). New York: Routledge.

Clarà, M. y Mauri, T. (2010). Una discusión sobre el conocimiento práctico y sus relaciones con el conocimiento teórico y la práctica. Infancia y Aprendizaje, 33(2), 199-207.

Cochran-Smith, M. y Lytle, S. (1999). Relationships of knowledge and practice: teacher learning in communities. Review of Research in Education, 24, 249-305.

Cochran-Smith, M. y Villegas, A. M. (2015). Studying teacher preparation: the questions that drive research. European Educational Research Journal, 14(5), 379-394.

Darling-Hammond, L. (2010). Teacher Education and the American Future. Journal of Teacher Education, 61(1-2), 35-47.

Darling-Hammond, L. (2017). Teacher education around the world: What can we learn from international practice? European Journal of Teacher Education, 40(3), 291-309.

Day, C. (2005). Formar docentes: cómo, cuándo y en qué condiciones aprende el profesorado. Madrid: Narcea.

Dotger, B. H. (2015). Core Pedagogy: Individual Uncertainty, Shared Practice, Formative Ethos. Journal of Teacher Education, 66(3), 215-226.

Domingo, A. (2013). Práctica reflexiva para docentes. De la reflexión ocasional a la reflexión metodológica. Alemania: Publicia.

Domingo, A. y Gómez, M. V. (2014). La práctica reflexiva: bases modelos e instrumentos. Madrid: Narcea.

Dufour, R. (2004). What is a "professional learning community"? Educational Leadership, 61 (8), 6-11.

Fleet, A., Patterson, C. y Robertson, J. (Eds.) (2017). Pedagogical documentation in early years practice. Seeing through multiple perpectives. London: SAGE Publications.

Flores, M. A. (2017). Practice, theory and research in initial teacher education: international perspectives. European Journal of Teacher Education, 40(3), 287-290.

Gessa, A. (2011). La coevaluación como metodología complementaria de la evaluación del aprendizaje. Análisis y reflexión en las aulas universitarias. Revista de Educación, 354, 749-764.

Glass, G. V. y Rud, A. G. (2012). The struggle between indiviualism and comunitarism: the pressure of population, prejudice, and the pursue. Review of Research in Education, 36, 95-112.

González, M. y Fuentes, E. J. (2011). El Prácticum en el aprendizaje de la profesión docente. Revista de Educación, 354, 47-70.

Green, W., Ruutz, A., Houghton, L. y Hibbins, R. (2016). Enabling Stories: Narrative, Leadership, Learning and Identity in a Faculty-Based Teaching Community of 
Practice. En J. McDonald y A. Cater-Steel (Eds.), Implementing Communities of Practice in Higher Education (pp. 159-181). Singapore: Springer.

Huber, J. Caine, V., Huber, M. y Steeves, P. (2013). Narrative Inquiry as Pedagogy in Education: The Extraordinary Potential of Living, Telling, Retelling, and Reliving Stories of Experience. Review of Research in Education, 37, 212-242.

Huber, G. L. y Gürtler, L. (2012). AQUAD. Manual del programa para analizar datos cualitativos (1. ed. 2003, Tübingen: Ingeborg Huber Verlag). Tübingen: Günter Huber.

Kelchtermans, G. (2014). Narrative-biographical pedagogies in teacher education. En C. Craig y L. Orland-Barak (Eds.), International Teacher Education: Promising Pedagogies (Part A) (pp. 273-291). London: Emerald Group Publishing Limited.

Kitchen, J. y Petrarca, D. (2016). Approaches to Teacher Education. En J. Loughram y M. L. Hamilton. International Handbook of Teacher Education. Volume 1. (pp. 137-186). Singapore: Springer.

Korthagen, F. (2010). La práctica, la teoría y la persona en la formación del profesorado. Revista Interuniversitaria de Formación del Profesorado, 68(24,2), 83-101.

Lieberman, A. y Pointer-Mace, D. H. (2008). Teacher Learning: the key to Educational Reform. Journal of Teacher Education, 59(3), 226-234.

Mena, J., Sánchez, E. y Tillema, H. H. (2011). Promoting teacher reflection: what is said to be done. Journal of Education for Teaching, 37(1), 21-36.

Payne, K. A. y Zeichner, K. (2017). Mutliple voice and participants in Teacher Education. En J. Clandinin y J. Husu. The Sage Handbook of Research on Teacher Vol. II (pp.1101-1116). Los Angeles: SAGE Reference.

Perrenoud, P. (2004). Desarrollar la práctica reflexiva en el oficio de enseñar. Barcelona: Graó.

Rodgers, C. y LaBoskey, V. K. (2016). Reflective Practice. En J. Loughram y M. L. Hamilton, International Handbook of Teacher Education. Volume 2 (pp. 71-104). Singapore: Springer.

Saiz, Á. y Susinos, T. (2017). Problemas pedagógicos para un Prácticum reflexive de maestros. Revista Complutense de Educación, 28(4), 993-1008.

Stenberg K., Karlsson, L., Pitkaniemi, H. y Maaranen, K. (2014). Beginning student teachers' teacher identities based on their practical theories. European Journal of Teacher Education, 37(2), 204-219. https://doi.org/10.1080/02619768.2014.882309.

Schön, D. A. (1992). La formación de profesionales reflexivos: Hacia un nuevo diseño de la enseñanza y el aprendizaje en las profesiones. Barcelona: Paidós.

Wenger, E. (2001). Comunidades de práctica: aprendizaje, significado e identidad. Barcelona: Paidós.

Williams, J. (2014). Teacher Educator Professional Learning in the Third Space Implications for Identity and Practice. Journal of Teacher Education, 65(4), 315-326. 
White, S. y Forgasz, R. (2016). The Practicum: The Place of Experience? En J. Loughram y M. L. Hamilton. International Handbook of Teacher Education. Volume 1 (pp. 231-266). Singapore: Springer.

Zabalza, M. A. (2016). El Prácticum y las prácticas externas en la formación universitaria. Revista Prácticum, 1(1), 1-23.

Zeichner, K. (2010). Nuevas epistemologías en formación del profesorado. Repensando las conexiones entre las asignaturas del campus y las experiencias de prácticas en la formación del profesorado en la universidad. Revista Iberoamericana de Formación del Profesorado, 68(24, 2), 123-149.

Zeichner, K. (2014). The struggle for the soul of teaching and teacher education in the USA. Journal of Education for Teaching, 40(5), 551-568. 